\title{
Migraine faciale à expression dentaire, à propos de trois cas
}

\author{
A. Ceddaha Zibi (Paris)
}

Migraine faciale à expression dentaire, à propos de trois cas. Anael CEDDAHA ZIBI1, Vanina LUCIANI2, Diane NGUYEN3, Audrey CHANLON3, Nathan MOREAU4,5 1 - Interne en chirurgie orale 2 - Etudiante en $6^{\text {ème }}$ année de chirurgie dentaire, Faculté de Chirurgie dentaire, Université Paris Descartes 3 - Praticien attaché, consultation de diagnostic et traitement des douleurs chroniques oro-faciales, service de médecine bucco-dentaire, Hôpital Bretonneau, AP-HP, Paris 4 - Responsable de la consultation de diagnostic et traitement des douleurs chroniques oro-faciales, service de médecine bucco-dentaire, Hôpital Bretonneau, AP-HP, Paris 5 - MCU-PH en médecine et chirurgie orale, Faculté de Chirurgie Dentaire, Université Paris Descartes \& Service de Médecine buccodentaire, Hôpital Bretonneau, AP-HP, Paris \& Laboratoire de Neurobiologie Oro-Faciale, Université Paris Diderot La migraine est une affection neurovasculaire qui peut être invalidante, sa pathogénie n'est pas encore parfaitement élucidée aujourd'hui. Le plus souvent à type de céphalée, elle représente la céphalée primaire la plus fréquente. Si son diagnostic est aisé lorsqu'elle se présente sous forme de céphalée, il devient plus délicat devant des manifestations faciales de migraines moins typiques. Les migraines avec une atteinte oro-faciale stricte sont peu décrites dans la littérature. Notre étude portera sur trois cas de patients recrutés et suivis à la consultation douleur de l'hôpital Bretonneau ayant souffert de douleurs oro- faciales, étiquetées comme migraines. Ce diagnostic ayant été confirmé par la suite lors de leurs prise en charge en neurologie. Dans le cas des migraines à expression dentaire, la maladie si elle est méconnue risque d'entraîner des traitements dentaires abusifs et inadaptés, comme cela a été le cas pour ces trois patientes. L'errance diagnostique et la chronicité des douleurs engendrent une dégradation de la qualité de vie des malades, et impacte souvent leur relation affective et professionnelle. L'aspect économique des traitements dentaires à répétition chez ces patients n'est pas à négliger. Si la migraine est une maladie bénigne elle peut devenir invalidante. La forte prévalence des migraines dans la population générale et dans la population active en fait une des priorités de santé publique du fait de son retentissement économique. Les objectifs de la prise en charge thérapeutique prennent en compte l'éradication des facteurs déclenchant des crises, le traitement de la crise migraineuse, et un traitement de fond prophylactique lorsque la fréquence des crises est importante. La migraine demeure une maladie sous-diagnostiquée dans toutes ses formes d'expression. Un diagnostic précoce demeure pourtant indispensable pour une prise en charge optimale en particulier en cas de manifestations oro-faciales pures. 